\title{
Commentary: The patient is the focus, but the data are the key: Toward data-driven critical care environments
}

\author{
Craig G. Rusin, PhD, ${ }^{a}$ Javier J. Lasa, MD, FAAP, ${ }^{a, b}$ and Paul A. Checchia, MD, FCCM, FAAC ${ }^{b}$
}

From the Divisions of ${ }^{\mathrm{a} C}$ ardiology and ${ }^{\mathrm{b}} \mathrm{Critical}$ Care Medicine, Department of Pediatrics, Baylor College of Medicine, Texas Children's Hospital, Houston, Tex.

Disclosures: Dr Rusin is the co-founder and CTO of Medical Informatics Corp. Neither he nor Medical Informatics Corp has a relationship to the authors of the original manuscript under consideration or to their institution. All other authors have nothing to disclose with regard to commercial support.

Received for publication Feb 26, 2019; accepted for publication Feb 26, 2019; available ahead of print April 6, 2019.

Address for reprints: Javier J. Lasa, MD, FAAP, Divisions of Critical Care Medicine and Cardiology, Department of Pediatrics, Baylor College of Medicine, Texas Children's Hospital, MC: E1420, 6651 Main St, Houston, TX 77030 (E-mail: jjlasa@texaschildrens.org).

J Thorac Cardiovasc Surg 2019;158:244-5

$0022-5223 / \$ 36.00$

Copyright (c) 2019 by The American Association for Thoracic Surgery

https://doi.org/10.1016/j.jtcvs.2019.02.105

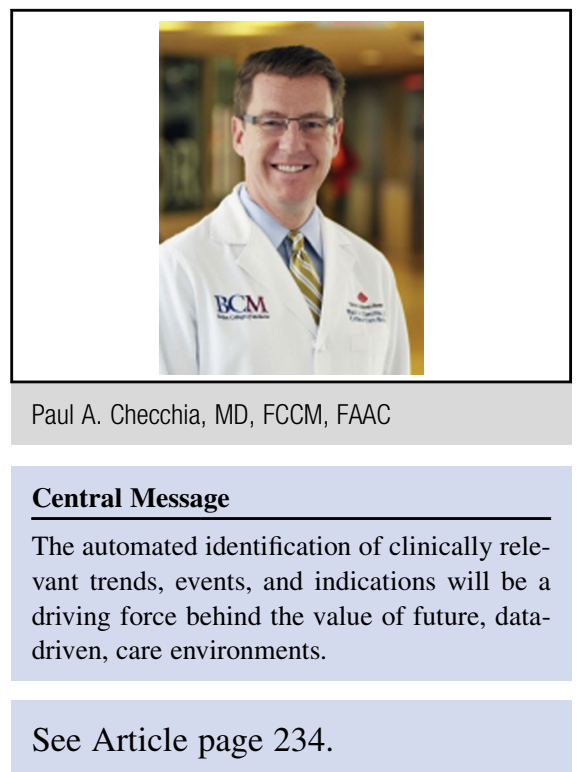

Physiologic monitoring is a central part of the care provided to patients during their stay in an intensive care unit. Such devices enhance the ability of care teams by alerting them to potentially harmful changes in the continuous stream of vital signs. Enormous amounts of data are generated in the process, often exceeding the amount of data contained within a patient's genome for each week of intensive care unit care. Although there is increased awareness that capturing patient data is valuable, there is less clarity around how to realize this value for the benefit of patients.

The automated identification of clinically relevant trends, events, and indications will be a driving force behind the value of future, data-driven, care environments. Ruiz and colleagues $^{1}$ demonstrate the beginning of this evolution. These researchers, along with others in the field, present a new way of enhancing the ability of care teams: Leveraging computers to continuously and automatically recognize clinical precursors that occur before acute patient decompensation and alert the team to their presence. This is especially important with complex patients such as children with single ventricle physiology where even in the best centers, sudden catastrophic events still occur frequently. Providing early warning alerts can allow care teams time to mitigate such events before they become life threatening.

Ruiz and colleagues ${ }^{1}$ confirm previous findings that there are subtle yet detectable changes in the status of patients with single ventricle physiology hours before deterioration occurs. ${ }^{2}$ Replication of this result provides evidence that such algorithms are identifying a real physiologic pattern. ${ }^{3}$ The challenge is in pairing such algorithms with effective clinical protocols to be enacted when computer-generated alerts are received. ${ }^{4}$ Additionally, variations in practice patterns across centers can potentially invalidate an algorithm that has only been trained on data from a single site. Multicenter studies focusing on outcomes are necessary to address these issues. Several such studies are in progress,

with at least 1 addressing patients with single-ventricle physiology.

Building a data-driven care environment will not be an easy task. It will require teams of clinical experts, data scientists, and engineers to work together effectively. It will also require a realignment of the traditional data science roles within institutions. Historically within academic hospitals, the role of data scientist was filled by a statistician and/or database administrator. Although traditional statistical and database support will still be necessary, institutional adoption of machine learning skills will become increasingly important as the capabilities of this field become more accessible to health care organizations. The multidisciplinary team represented by Ruiz and colleagues ${ }^{1}$ provides a potential model for how effective partnerships can be achieved. The end result will be the development of technologies that provide smarter, safer, and more effective clinical environments, allowing even the best care teams to improve the quality of the care they provide to patients.

\section{References}

1. Ruiz VM, Saenz L, Lopez-Magallon A, Shields A, Ogoe HA, Suresh S, et al. Early prediction of critical events for infants with single-ventricle physiology in critical care using routinely collected data. J Thorac Cardiovasc Surg. 2019; 158:234-43.e3. 2. Rusin CC, Acosta SI, Shekerdemian LS, Vu EL, Bavare AC, Myers RB, et al. Prediction of imminent, severe deterioration of children with parallel circulations 
using real-time processing of physiologic data. J Thorac Cardiovasc Surg. 2016; $152: 171-7$.

3. Vu EL, Rusin CG, Penny DJ, Kibler KK, Easley RB, Smith B, et al. A novel electrocardiogram algorithm utilizing ST-segment instability for detection of cardiopulmonary arrest in single ventricle physiology: a retrospective study. Pediatr Crit Care Med. 2017;18:44-53.

4. Kennedy CE, Aoki N, Mariscalco M, Turley JP. Using time series analysis to predict cardiac arrest in a PICU. Pediatr Crit Care Med. 2015;16:e332-9. 